\title{
$v f r$, A Global Regulatory Gene, is Required for Pyrrolnitrin but not for Phenazine-1-carboxylic Acid Biosynthesis in Pseudomonas chlororaphis G05
}

\author{
Xia Wu ${ }^{1 \dagger}$, Xiaoyan $\mathrm{Chi}^{1 \dagger}$, Yanhua Wang ${ }^{1 \dagger}$, Kailu Zhang ${ }^{1}$, Le Kai ${ }^{1}$, Qiuning He ${ }^{1}$, Jinxiu Tang', Kewen Wang', \\ Longshuo Sun ${ }^{1}$, Xiuying $\mathrm{Hao}^{2}$, Weihai Xie (iD) ${ }^{1 *}$, and Yihe Ge (iD) ${ }^{1 *}$ \\ ${ }^{l}$ Department of Applied and Environmental Microbiology, School of Life Sciences, Ludong University, Yantai 264025, \\ China \\ ${ }^{2}$ Institute of Applied Microbiology, Xinjiang Academy of Agricultural Sciences, Urumqi 830001, China
}

(Received on January 16, 2019; Revised on April 7, 2019; Accepted on April 9, 2019)

In our previous study, pyrrolnitrin produced in Pseudomonas chlororaphis G05 plays more critical role in suppression of mycelial growth of some fungal pathogens that cause plant diseases in agriculture. Although some regulators for pyrrolnitrin biosynthesis were identified, the pyrrolnitrin regulation pathway was not fully constructed. During our screening novel regulator candidates, we obtained a white conjugant G05W02 while transposon mutagenesis was carried out between a fusion mutant G05 $\Delta p h z \Delta p r n:: l a c Z$ and $E$. coli S171 (pUT/mini-Tn5Kan). By cloning and sequencing of the transposon-flanking DNA fragment, we found that a $v f r$ gene in the conjugant G05W02 was disrupted with mini-Tn5Kan. In one other previous study on $\boldsymbol{P}$. fluorescens, however, it was reported that the deletion of the $v f r$ caused increased production of pyrrolnitrin and other antifungal metabolites. To confirm its regulatory function, we constructed the $v f r$-knockout mutant G05 $\Delta v f r$ and G05 $\Delta p h z \Delta p r n:: l a c Z \Delta v f r$. By quantifying

\footnotetext{
${ }^{\top}$ These authors contributed this work equally.

*Co-corresponding authors.

Weihai Xie

Phone) +86-535-6681053, FAX) +86-535-6692760

E-mail)xieweihai@ldu.edu.cn

ORCID

https://orcid.org/0000-0002-6602-3075

Yihe Ge

Phone) +86-535-6685003, FAX) +86-535-6692726

E-mail)geyihe@1du.edu.cn

https://orcid.org/0000-0002-7938-8292

(c) This is an Open Access article distributed under the terms of the Creative Commons Attribution Non-Commercial License (http:// creativecommons.org/licenses/by-nc/4.0) which permits unrestricted noncommercial use, distribution, and reproduction in any medium, provided the original work is properly cited.
}

Articles can be freely viewed online at www.ppjonline.org. $\beta$-galactosidase activities, we found that deletion of the $v f r$ decreased the prn operon expression dramatically. Meanwhile, by quantifying pyrrolnitrin production in the mutant G05 $\Delta v f r$, we found that deficiency of the $\mathrm{Vfr}$ caused decreased pyrrolnitrin production. However, production of phenazine-1-carboxylic acid was same to that in the wild-type strain G05. Taken together, Vfr is required for pyrrolnitrin but not for phenazine-1-carboxylic acid biosynthesis in $P$. chlororaphis G05.

Keywords : P. chlororaphis, phenazine-1-carboxylic acid, pyrrolnitrin, regulation, $\mathrm{Vfr}$

Handling Editor : Sang, Mee Kyung

Now, some soil-borne fungal pathogens often cause diseases that lead to heavy yield losses in agriculture (Haas and Keel, 2003). Although some fungicides are effectively employed in protecting crops, their intensive applications are not permitted duo to concern for the environment and public health (Chen et al., 2018; D'Mello et al., 1998). Therefore, more and more fluorescent Pseudomonas sp. are paid great attention because they can alleviate plant diseases and increase crop productivity (Baehler et al., 2005; Haas and Defago, 2005; Haas and Keel, 2003). Pseudomonas chlororaphis $\mathrm{G} 05$ is a root-colonizing biocontrol agent that bioprotects some plants from the diseases caused by fungal phytopathogens, such as Fusarium oxysporum, Rhizoctonia solani, and F. graminearum (Chi et al., 2017; Ge et al., 2008; Huang et al., 2018). It has been demonstrated that antifungal compounds, phenazine-1-carboxylic acid and pyrrolnitrin that are produced in this bacterium, mainly contribute to suppression of mycelial growth of these phytopathogenic fungi (Chi et al., 2017; Huang et al., 2018). 
Up to date, besides phenazines and pyrrolnitrin, more and more antifungal compounds, including pyoleuteorin (PLT), hydrogen cyanide (HCN), 2,4-diacetylphloroglucinol (DAPG), lipopeptides, furanomycin and so on, have been identified in Pseudomonads' strains and exhibited a remarkable biocontrol ability (Fenton et al., 1992; Ge et al., 2004; Laville et al., 1992; Mavrodi et al., 1998; Thomashow and Weller, 1988; Trippe et al., 2013; Voisard et al., 1989).

In our previous study, we found that pyrrolnitrin played a more essential role than phenazines in growth suppression of $F$. graminearum and bioprotection of wheat crops against Fusarium head blight (FHB) disease (Huang et al.,
2018). The production of pyrrolnitrin, however, is not high in the wild-type strain G05. Therefore, to increase pyrrolnitrin production and expand its application in agriculture, we should screen and identify more novel regulators and create regulatory pathway of pyrrolnitrin in detail. In P. chlororaphis PA23, ANR and PtrA were identified to mediate pyrrolnitrin production (Nandi et al., 2016; Shah et al., 2016). In $P$. fluorescens FD6, RetS and Vfr were reported to regulate pyrrolnitrin biosynthesis (Zhang et al., 2015, 2016). In P. chlororaphis O6, RpoS and GacS deficiency could change the production of pyrrolnitrin (Oh et al., 2013; Park et al., 2018). Although pyrrolnitrin can be biosynthesized in many different genera of bacteria and some regulators

Table 1. Bacterial strains and plasmids used in this study

\begin{tabular}{|c|c|c|}
\hline Strain and plasmid & Relevant characteristics & Source/reference \\
\hline \multicolumn{3}{|l|}{ Strains } \\
\hline \multicolumn{3}{|l|}{ E. coli } \\
\hline $\mathrm{DH} 5 \alpha$ & Ф80 lacZAM15 $\Delta$ (lacZYA-argF) U169 hsdR17 recAlendA1 thi ${ }^{-} 1$ & Lab collection \\
\hline $\operatorname{SM10}(\lambda v f r)$ & $\mathrm{F}^{-}$thi ${ }^{-} 1$ thr 1 leuB6 recA tonA21 lacY1 supE44 $\left(\mathrm{Mu}_{\mathrm{C}}^{+}\right) \lambda$ pir $\mathrm{Kan}^{\mathrm{R}}$ & Lab collection \\
\hline \multicolumn{3}{|l|}{ P. chlororaphis } \\
\hline G05 & $\begin{array}{l}\text { The wild-type strain, phenazine-1-carboxylic acid and pyrrolnitrin producer, } \mathrm{PCA}^{+} \text {, } \\
\mathrm{PRN}^{+}, \mathrm{Spe}^{\mathrm{R}}\end{array}$ & Lab collection \\
\hline $\mathrm{G} 05 \Delta p h z \Delta p r n:: l a c Z$ & $\begin{array}{l}\text { The } p h z A B C D E F G \text { and } p r n A B C D \text { operons deleted and the } p r n A^{\prime} \text { fused with the } \\
\text { truncated } l a c Z \text { gene in frame in the wild-type strain } \mathrm{G} 05, \mathrm{Spe}^{\mathrm{R}} \mathrm{Gen}^{\mathrm{R}}\end{array}$ & Luo et al., 2018 \\
\hline $\mathrm{G} 05 \Delta v f r$ & $\begin{array}{l}\text { The } v f r \text { deleted and inserted with gentamicin resistance cassette in the wild-type } \\
\text { strain G05, } \operatorname{Spe}^{\mathrm{R}} \mathrm{Gen}^{\mathrm{R}}\end{array}$ & This study \\
\hline G05W02 & $\begin{array}{l}\text { A white conjugant isolated on LB plates by transposon random insertion on the } \\
\text { chromosome of the fusion mutant G05 } \Delta \text { phz } \Delta p r n:: l a c Z, \mathrm{Spe}^{\mathrm{R}} \mathrm{Kan}^{\mathrm{R}}\end{array}$ & This study \\
\hline $\mathrm{G} 05 \Delta p h z \Delta p r n:: l a c Z \Delta v f r$ & The $v f r$ deleted in the fusion mutant G05 $\Delta p h z \Delta p r n:: l a c Z, \operatorname{Spe}^{\mathrm{R}} \mathrm{Gen}^{\mathrm{R}}$ & This study \\
\hline \multicolumn{3}{|l|}{ Plasmids } \\
\hline pUCm-T & T-vector, ColE, Amp ${ }^{\mathrm{R}}$ & Sangon \\
\hline pUCTW02 & $\begin{array}{l}\text { Transposon-flanking DNA fragment amplified by inverse PCR cloned into pUCm-T, } \\
A m p^{R}\end{array}$ & This study \\
\hline $\mathrm{pEX} 18 \mathrm{Tc}$ & Gene replacement vector with MCS from pUC18, oriT ${ }^{+} a c B^{+}$, Tet $^{R}$ & Hoang et al., 1998 \\
\hline $\mathrm{pEXV}$ & pEX18Tc containing a $2.0 \mathrm{~kb} v f r$-flanking PCR fragment, Tet $^{\mathrm{R}}$ & This study \\
\hline pEXVG & $\begin{array}{l}\text { A } 0.8 \mathrm{~kb} X b a \mathrm{I} \text {-digested } a a c C 1 \text { fragment (gentamicin resistance cassette) inserted in } \\
X b a \mathrm{I} \text { site in } \mathrm{pEXV}, \operatorname{Tet}^{\mathrm{R}} \mathrm{Gen}^{\mathrm{R}}\end{array}$ & This study \\
\hline pME6010 & Low-copy shuttle vector between $E$. coli and Pseudomonas spp., Tet ${ }^{\mathrm{R}}$ & Heeb et al., 2000 \\
\hline pME10V & A $1.2 \mathrm{~kb} v f r$ amplified by PCR cloned in pME6010, Tet $^{\mathrm{R}}$ & This study \\
\hline pME6015 & Pvs1-p15A shuttle vector for translational lacZ fusion, Tet $^{\mathrm{R}}$ & Heeb et al., 2000 \\
\hline pME15N & $\begin{array}{l}\text { A } 0.9 \mathrm{~kb} \text { DNA DNA fragment containing the promoter region and the first } 10 \text { condon } \\
\text { of } p r n A \text { cloned in pME } 6015, \mathrm{Tet}^{\mathrm{R}}\end{array}$ & Zhang et al., 2018 \\
\hline pME15Z & $\begin{array}{l}\text { A } 0.9 \mathrm{~kb} \text { DNA DNA fragment containing the promoter region and the first } 8 \text { condon } \\
\text { of } p h z A \text { cloned in pME } 6015, \text { Tet }^{\mathrm{R}}\end{array}$ & Zhang et al., 2018 \\
\hline pME6522 & pVS1-p15A shuttle vector for transcriptional lacZ fusion and promoter probing, Tet $^{\mathrm{R}}$ & Blumer et al., 1999 \\
\hline pME22N & $\begin{array}{l}\text { pME6522 carrying a } 0.8 \mathrm{~kb} \text { upstream region of prn (promoter region) and transcrip- } \\
\text { tional fusion prnA-lacZ, } \mathrm{Tet}^{\mathrm{R}}\end{array}$ & Zhang et al., 2018 \\
\hline pME22Z & $\begin{array}{l}\text { pME6522 carrying a } 0.8 \mathrm{~kb} \text { upstream region of } p h z \text { (promoter region) and transcrip- } \\
\text { tional fusion phzA1-lacZ, } \text { Tet }^{\mathrm{R}}\end{array}$ & Zhang et al., 2018 \\
\hline pUCGm & Gentamicin resistance gene cassette $(a a c C 1)$ resource, cloning vector, $\mathrm{Amp}^{\mathrm{R}} \mathrm{Gen}^{\mathrm{R}}$ & Schweizer, 1993 \\
\hline
\end{tabular}


Table 2. Oligonucleotide primers used in this study

\begin{tabular}{|c|c|}
\hline Primers & Sequences $\left(5^{\prime}-3^{\prime}\right.$, artificial restriction enzyme site was underlined) \\
\hline $\mathrm{V}-1 \mathrm{~F}$ & CAGCGACAAGGTCAGCCTGGGTTTC \\
\hline $\mathrm{V}-1 \mathrm{R}_{\mathrm{Xba}}$ & CATGATTCTAGATAGCGGCGGCGCTGGCAGTGCATC (XbaI) \\
\hline $\mathrm{V}-2 \mathrm{~F}_{\mathrm{Xba}}$ & GATCATTCTAGACCTCGAAGAACGCAACCTGGTCC (XbaI) \\
\hline $\mathrm{V}-2 \mathrm{R}$ & CGTGCTGTTGATTGTGGCGGCGCTG \\
\hline $\mathrm{V}-3 \mathrm{~F}_{\mathrm{Acc}}$ & CAAGTTGGTACCCGGGCGATTCTCGAGCAGATGCG (Acc65I) \\
\hline $\mathrm{V}-3 \mathrm{R}_{\text {Hin }}$ & 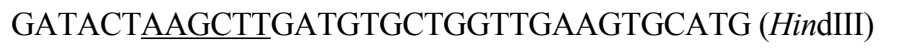 \\
\hline G-F & GCAGCAACGATGTTACGCAG \\
\hline G-R & TGTTAGGTGGCGGTACTTGG \\
\hline G-LF & GTCACAACGCCGCGGCCAATTC \\
\hline G-LR & CAGGCTTATGTCAATTCGAGCTC \\
\hline $\mathrm{V}-\mathrm{WF}_{\mathrm{Eco}}$ & GAACTTGAATTCAGGATGCTGACCACGTCGAAG $(E c o$ RI) \\
\hline $\mathrm{V}-\mathrm{WR}$ Xho & CAAGTTCTCGAGCGGGAAACCATGGTCGCGGCG $(X h o \mathrm{I})$ \\
\hline TN5-inF & CGCTCCCGATTCGCAGCGCATCGCC \\
\hline TN5-inR & CCAAGCGGCCGGAGAACCTGCGTGC \\
\hline M13-F & GTTGTAAAACGACGGCCAG \\
\hline M13-R & CAGGAAACAGCTATGAC \\
\hline RT-rpoDF & GTGGTCGTGAGCAGGGTTACCTGAC \\
\hline RT-rpoDR & GGATGATGTCTTCCACCTGTTCCGG \\
\hline RT-prnAF & CAGCAGCAAGCGAACATTACGCTC \\
\hline RT-prnAR & CGGTATCCCGAGGAAGTCGAAGAAC \\
\hline
\end{tabular}

that mediate its biosynthesis have been identified, its regulatory pathway in detail is not fully made clear. To identify more novel regulatory candidate genes involving in pyrrolnitrin biosynthesis, in our study with $P$. chlororaphis G05, We first constructed the fusion mutant G05 $\Delta p h z \Delta p r n:: l a c Z$ (Luo et al., 2018). In this mutant, the $p h z$ operon (phz$A B C D E F G$, phenazine biosynthetic loci) was knocked out and the prn operon (prnABCD, pyrrolnitrin biosynthetic loci) was deleted and its promoter zone was in-frame fused with the truncated lacZ reporter gene (Minton, 1984). With the fusion mutant G05 pphz $\Delta p r n:: l a c Z$ as recipient cell, conjugation mating was then carried out with random insertion of transposonMini-Tn5Kan (de Lorenzo et al., 1990). One white colony was fortunately found and isolated in an LB agar plate supplemented with 5-bromo-4-chloro-3-indolyl $\beta$-D-galactopyranoside (X-gal). By inverse PCR, we cloned and identified the site of transposon insertion. $v f r$, a novel candidate gene mediating the pyrrolnitrin biosynthesis was then identified. In this study, we confirmed that $v f r$ was indeed required for pyrrolnitrin, but not for phenazine1-carboxylic acid biosynthesis in P. chlororaphis G05.

\section{Materials and Methods}

Bacterial strains, plasmids, primers and culture conditions. All strains and plasmids employed in this work are listed in Table 1. All oligonucleotide primers used for regu- lar PCRs or RT-qPCRs in this study are showed in Table 2. Escherichia coli strains were routinely cultivated in LuriaBertani (LB) medium at $37^{\circ} \mathrm{C}$ (Sambrook and Russell, 2001). P. chlororaphis strains were regularly grown in LB medium at $30^{\circ} \mathrm{C}$ (Ge et al., 2008), or in glycerol-alanine medium (GA) at $30^{\circ} \mathrm{C}$ for phenazine assays (Chieda et al., 2005). If required, ampicillin (Amp, $100 \mu \mathrm{g} / \mathrm{ml}$ ), chloramphenicol (Chl, $30 \mu \mathrm{g} / \mathrm{ml}$ ), spectinomycin (Spe, $100 \mu \mathrm{g} / \mathrm{ml}$ ), kanamycin (Kan, $50 \mu \mathrm{g} / \mathrm{ml}$ ), and gentamicin (Gen, $20 \mu \mathrm{g} /$ $\mathrm{ml})$ were supplemented in medium for $E$. coli growth. For $P$. chlororaphis growth, tetracycline (Tet, $125 \mu \mathrm{g} / \mathrm{ml}$ ), gentamicin $(40 \mu \mathrm{g} / \mathrm{ml})$ were used in its medium.

Recombinant DNA techniques. Standard techniques were employed for gel electrophoresis, restriction endonuclease digestion, and ligation (Sambrook and Russell, 2001). Plasmid DNA isolation from E. coli and P. chlororaphis strains was carried out with alkaline lysis method or with the recommended protocols provided by Plasmid DNA Extraction Kit (Sangon, Shanghai, China). Chromosomal DNA was isolated from $P$. chlororaphis using the Genomic DNA Extraction Kit (Solarbio, Beijing, China) or the regular method as described by Chen and Kuo (1993). Regular PCR amplifications were carried out with a $25 \mu$ reaction mixture containing $1 \times \mathrm{LA}$ with $\mathrm{GC}$ buffer, $2 \mathrm{mM} \mathrm{MgSO}_{4}$, $200 \mu \mathrm{M}$ (each) dATP, dGTP, dCTP, and dTTP, $10 \mathrm{pmol}$ of each primer, $0.2 \mu \mathrm{LA}$ DNA polymerase (Takara Bio, 
Dalian, China), and $10 \mathrm{ng}$ of purified genomic DNA of the strain G05 or its derivative mutants. All the amplifications were performed in $\mathrm{T} 100^{\mathrm{TM}}$ thermal cycler (Bio-Rad Laboratory, Hercules, CA, USA). The cycling program started with a 2-min pre-denaturation at $94^{\circ} \mathrm{C}$, followed by $33 \mathrm{cy}-$ cles $\left(30\right.$-sec denaturation at $94^{\circ} \mathrm{C}, 30 \mathrm{~s}$ anneal at $60-66^{\circ} \mathrm{C}$, 2-min extension at $72^{\circ} \mathrm{C}$ ), and ended with 7 -min final extension at $72^{\circ} \mathrm{C}$. PCR amplicons were routinely purified using PCR Purification Kit (Sangon, Shanghai, China). To do transformation, $P$. chlororaphis competent cells were first prepared and electroporation was then performed as described by Smith and Iglewski (1989).

Transposon mutagenesis and identification of transposon localization. Random mutagenesis was performed using transposon mini-Tn5Kan which contains a kanamycin resistance marker (de Lorenzo et al., 1990). Bacterial conjugations were carried out to introduce mini-Tn5Kan into the $P$. chlororaphis chromosome. Briefly, a $500 \mu \mathrm{l}$ sample of each of two overnight cultures, E. coli S17-1( $\lambda$ pir) /pUT/

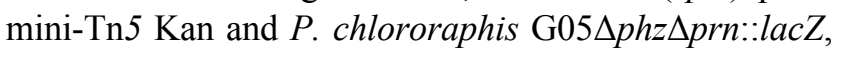
was harvested, washed twice with LB medium, mixed together into a $100 \mu \mathrm{l}$ aliquot, then transferred onto a $25-\mathrm{mm}$ diameter filter $(0.22 \mu \mathrm{m}$ pore size $)$ that was placed on the surface of an LB agar plate, and grown for at least $12 \mathrm{~h}$ at $30^{\circ} \mathrm{C}$. The cells grown on the filter surface were then suspended in $1 \mathrm{ml}$ of LB broth, diluted and spread on LB agar plates that contained Kanamycin, chloramphenicol, and X-gal. Plates were kept in an incubator at $30^{\circ} \mathrm{C}$ till blue colonies developed. A white colony named G05W02 developed around many blue colonies after 3 days of growth, and was then isolated.

To identify the localization of transposon insertion, chromosomal DNA from the white conjugant G05W02 was isolated, digested with SalI, purified, and self-ligated. With the purified ligation as a template, inverse PCR amplification was then carried out using a pair of primers TN5-inF and TN5-inR. The PCR amplicons were finally cloned into pUCm-T (T-vector), and sequenced using primers M13-F and M13-R.

Construction of the $v f r$ knockout mutant with homologous recombination. To confirm the $v f r$ function in $P$. chlororaphis $\mathrm{G} 05$, we constructed a $v$ fr-defective mutant G05 $v v f r$ using a homologous recombination strategy (Hoang et al., 1998), in which the $v$ fr DNA region was deleted and replaced with the gentamicin resistance genes ( $a a c C 1)$ in chromosome. Firstly, two PCR amplifications were performed with two pairs of primers $\left(\mathrm{V}-1 \mathrm{~F} / \mathrm{V}-1 \mathrm{R}_{\mathrm{Xba}}\right.$ and $\mathrm{V}$ $2 \mathrm{~F}_{\mathrm{Xba}} / \mathrm{V}-2 \mathrm{R}$ ), obtaining two $1.0 \mathrm{~kb}$ amplicons: one is a 1007 bp portion of the G05 genome upstream of the $v f r$; another is a $1100 \mathrm{bp}$ region downstream of the $v f r$. Two amplicons were pooled, purified, digested with $X b a \mathrm{I}$, re-purified, and finally ligated. The purified ligation was used as the template and the nested PCR was performed with a pair of primers $\left(\mathrm{V}-3 \mathrm{~F}_{\mathrm{Acc}} / \mathrm{V}-3 \mathrm{R}_{\mathrm{Hin}}\right)$ to obtain $2.0 \mathrm{~kb}$ PCR products. After simultaneous digestion with Acc65I and HindIII, the digested PCR products were cloned into the suicide plasmid pEX18Tc, resulting in pEXV (Hoang et al., 1998). Secondly, an 878 bp gentamicin resistance gene (aacC1) was purified with $X b a \mathrm{I}$-digestion of pUCGm (Schweizer, 1993), then inserted into the $X b a \mathrm{I}$ site in pEXV to yield pEXVG.

After sequence confirmation, biparental mating was carried out and the derivative pEXVG was mobilized to $P$. chlororaphis $\mathrm{G} 05$ from $E$. coli SM10. The potential mutant G05 $\Delta v f r$ was screened and isolated on LB medium plates supplemented with $10 \%$ sucrose and gentamicin, suggesting that a double-crossover event had occurred (Ge et al., 2007; Hoang et al., 1998). In addition, biparental mating was also performed between E. coli SM10/ pEXV and G05 $\Delta$ phz $\Delta p r n:$ :lacZ, generating the mutant G05 $\Delta h z \Delta p r n:: l a c Z \Delta v f r$. All mutants were verified by PCR using the primers G-F/G-R and G-LF/G-LR that annealed in the gentamicin resistance cassette specifically (data now shown).

Construction of the $v f r$ expression vector for complementation assay. To complement the mutant G05 $\Delta v f r$, pME10V was constructed as follows. The $1.0 \mathrm{~kb}$ DNA amplicons containing the whole $v$ fr amplified by PCR with primers $\mathrm{V}-\mathrm{WF}_{\mathrm{Eco}}$ and $\mathrm{V}-\mathrm{WR}_{\mathrm{Xho}}$ were cleaved with EcoRI and $X h o \mathrm{I}$, and then cloned into the same sites of a lowcopy shuttle vector pME6010, creating pME10V (Heeb et al., 2000). After sequence confirmation, pME10V and pME6010 were respectively transformed into G05 $v$ fr and other derivatives for complementation assay.

RNA extraction and real-time quantitative PCR (RTqPCR). Pseudomonas strains were cultivated in GA broth similarly to genomic DNA preparation. The prnA was selected for qRT-PCR analysis. Cells grown for $24 \mathrm{~h}, 48 \mathrm{~h}$, and $72 \mathrm{~h}$ were havested. The total RNAs was isolated from cells of the strain G05 and G05 $\Delta v f r$ using a TRIzol reagent (Takara, Dailian, China) according to manufacturer's instructions. The trace of genomic DNA in total RNA samples was removed with digestion using RNase-free DNase I. Reverse transcription to cDNA was performed at $42^{\circ} \mathrm{C}$ for 60 min using random hexamer primer with a RevertAid First Strand cDNA Synthesis Kit (Thermo Scientific). The 
resulting cDNA was amplified and quantified by RT-qPCR with a ChamQ ${ }^{\mathrm{TM}}$ SYBR qPCR Master Mix (Vazyme) on ABI $\mathrm{Q}_{6}$ Flex PCR system. The rpoD gene was used as a reference (Liu et al., 2018; Mulet et al., 2009). The primers RT-prnAF/RT-prnAR were designed to amplify 125bp DNA fragment in $p r n A$. The qPCR amplifications were carried out at $95^{\circ} \mathrm{C}$ for $30 \mathrm{~s}$, followed by 40 cycles of $95^{\circ} \mathrm{C}$ for $10 \mathrm{~s}$, and $58^{\circ} \mathrm{C}$ for $30 \mathrm{~s}$, and a final dissociation curve analysis step from 58 to $95^{\circ} \mathrm{C}$. The transcriptional level of prnA between G05 and G05 vvfr was compared by the $2^{-\Delta \Delta C \mathrm{t}}$ method (Livak and Schmittgen, 2001).

Phenazine-1-carboxylic acid assay. To quantify phenazine-1-carboxylic acid, the wild-type strain G05 and its derivatives were respectively inoculated in $150 \mathrm{ml} \mathrm{GA}$ broth at $30^{\circ} \mathrm{C}$ for $72 \mathrm{~h}$. Samples of each cultures were collected and quantified once every $12 \mathrm{~h}$. Samples were prepared with previously established methods and PCA was quantified spectrophotometrically at $252 \mathrm{~nm}$ (Cui et al., 2016; Kim, 2000).

Pyrrolnitrin assay. To quantify pyrrolnitrin, bacterial strains were cultivated with same methods above. Samples were prepared with previously created methods (Huang et al., 2018). Pyrrolnitrin quantified by high performance liquid chromatography (HPLC) with reverse phase C18 column (Ovadis et al., 2004). Standard sample of pyrrolnitrin was purchased from Sigma-Aldrich (St. Louis, MO, the U.S.A.).

$\beta$-Galactosidase activity assay. For $\beta$-galactosidase enzyme assay, the wild-type strain G05 and its derivative were grown in $150 \mathrm{ml}$ of GA or LB medium at $30^{\circ} \mathrm{C}$. Samples were harvested after a specified period of growth. After treated with SDS and chloroform in appropriate amounts, $\beta$-galactosidase activities were released and quantified with standard methods (Miller, 1972).

Statistical analysis. All statistical data in this work were analyzed and processed with an analysis of variance test (ANOVA) or a two-tailed paired Student $t$-test using the statistical software package SPSS (Chicago, IL, USA), and Duncan's multiple range test was employed for means separation of antifungal compound production and $\beta$-galactosidase activities. Values of $P<0.05$ were considered statistically significant, and values of $P<0.01$ were extremely significant.

Nucleic sequence accession number. The $v$ fr gene sequence was deposited in GenBank and accession number was assigned with MK288018.

\section{Results}

Isolation and characterization of the blue-changed mutant G05W02. To identify more novel regulators that modulate the prn expression, mini-Tn5-mediated mutagenesis was carried out between $E$. coli and $P$. chlororaphis G05 $\Delta p h z \Delta p r n:: l a c Z$. In an LB medium plate containing $\mathrm{X}$-gal and kanamycin, a white colony, called G05W02, was screened and picked up. To confirm its color change and mutagenesis, we streaked it in another X-gal-supplemented LB medium plate again, using its parental strain G05 phz $\Delta p r n:: l a c Z$ as a control. As shown in Fig. 1A,
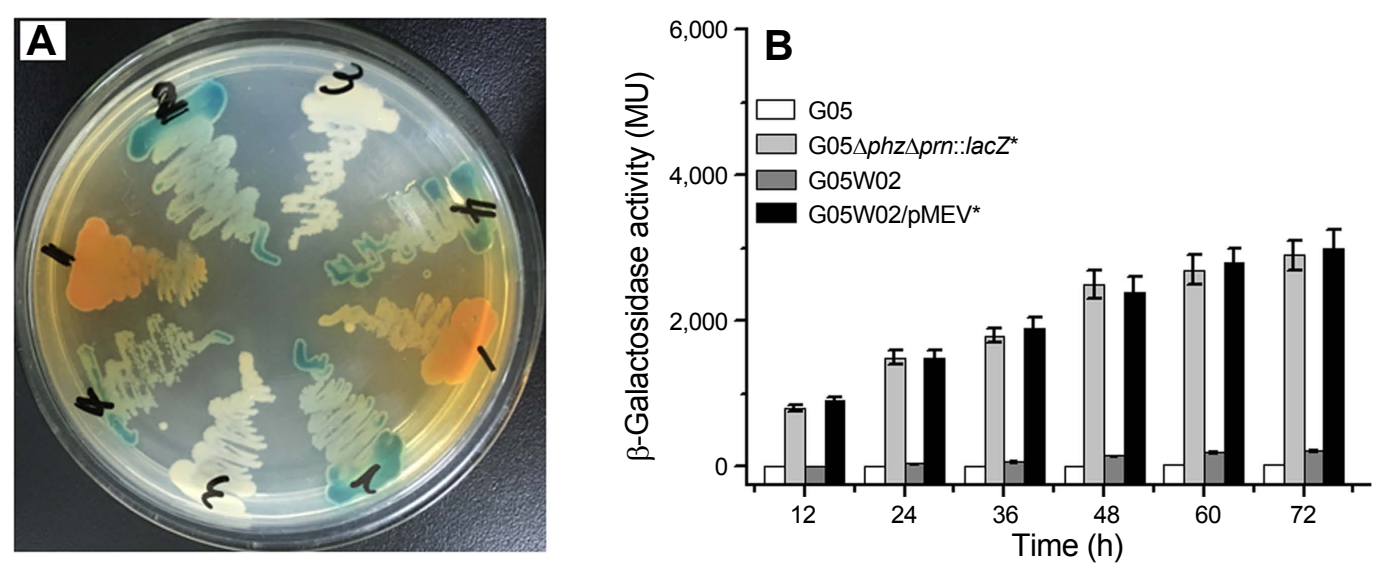

Fig. 1. Characterizations of the conjugant G05W02 and its derivatives. (A) Color of colonies shown in the LB medium supplemented with X-gal. Arabic numbers from 1 to 4 stand for the wild-type strain G05, the fusion mutant G05 $\Delta$ phz $\Delta p r n:$ lacZ, the transposon mutant G05W02, and the transformant G05W02/pME10V, respectively. (B) $\beta$-Galactosidase activities were quantified when they were grown in GA medium at $30^{\circ} \mathrm{C}$ for $72 \mathrm{~h}$. The values from three independent experiments were presented as the average $\pm s t a n d a r d ~ d e v i a t i o n$. Superscript of asterisk followed the strains indicated no significant differences $(P>0.05)$. 
the exconjugant G05W02 totally differed from its parental strain G05 $\Delta$ hz $\Delta p r n:: l a c Z$ with white color. Meanwhile, we quantified its $\beta$-galactosidase activities while it grown in GA medium for $72 \mathrm{~h}$. As shown in Fig. 1B, in

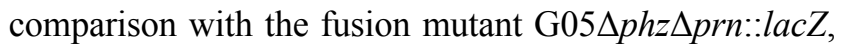
$\beta$-galactosidase activities produced by the transposonmediated mutant G05W02 were extremely low, suggesting that the expression of the prn operon was suppressed in this white exconjugant.

Localization of transposon insertion and identification of the $v f r$. To clone the flanking DNA fragment of transposon insertion, we employed inverse-PCR to amplify and identify the transposon-disrupted gene. Before PCR, the template of the genomic DNA of the conjugant G05W02 was prepared as described in Material and methods. After inverse PCR, $3.0 \mathrm{~kb}$ amplicon was cloned into the pUCm-T (T-vector) and created pUCTW02 for sequencing. Sequencing results verified that the transposon miniTn5Kan was actually inserted the $v f r$ gene in the conjugant G05W02. According to the sequence of the $v f r$, the predicted Vfr in the strain P. chlororaphis G05 contains 214 amino acid residues with a molecular mass of $24 \mathrm{KDa}$, showing closest similarity to that in the strain P. chlororaphis (99\%), P. fluorescens (98\%), P. aeruginosa PAO1 (83\%), and E. coli $\mathrm{K} 12$ (63\%).
Deletion of the $v f r$ caused decreased expression of the prn operon. To examine regulatory effects of Vfr on the expression of the prn operon, we first created the $v f r$ knockout mutant G05 $\Delta p h z \Delta p r n:$ :lacZ $\Delta v f r$. As shown in Fig. 2A, the mutant G05 $\Delta p h z \Delta p r n:: l a c Z \Delta v$ fr turned out to be white on a LB medium plate supplemented with X-gal. As it was complemented with bearing the shuttle plasmid pME10V, the transformant could turn blue again. The transformant harboring the original plasmid pME6010, however, did not turn blue. Meanwhile, we inoculated the fusion mutant G05 $\Delta p h z \Delta p r n:: l a c Z$ and its derivatives in GA medium, and then quantified their $\beta$-galactosidase activities. As shown in Fig. 2B, $\beta$-galactosidase activities produced in the $v f r$-knockout mutant G05 $\Delta p h z \Delta p r n:: l a c Z \Delta v f r$ were much lower than those in the parental strain G05 $\Delta p h z \Delta p r n:: l a c Z$. When the mutated $v f r$ gene was complemented with introduction of pME10V, however, the transformant G05 $\Delta p h z \Delta p r n:: l a c Z \Delta v f r / p M E 10 \mathrm{~V}$ produced almost same $\beta$-galactosidase activities as the parental strain $\mathrm{G} 05 \Delta p h z \Delta p r n:: l a c Z$ did. In addition, we also found that the

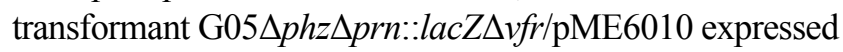
same $\beta$-galactosidase activities as the $v f r$-knockout mutant G05 $\Delta p h z \Delta p r n:: l a c Z \Delta v$ fr did.

These results indicated that the expression of the prn operon was indeed decreased in the absence of the $v f r$ gene, suggesting that the expression of the prn operon requires the presence of Vfr in the wild-type strain G05.
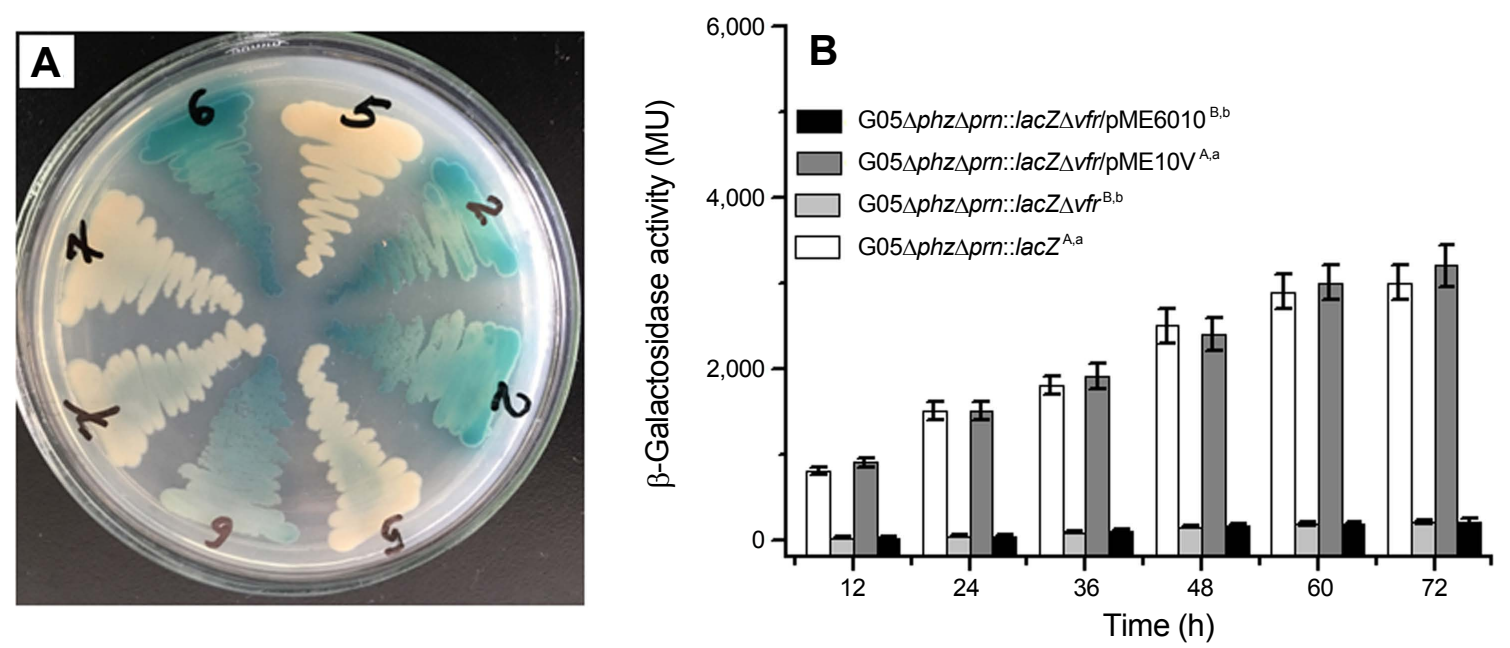

Fig. 2. Characterizations of the site-directed knockout mutant G05 $\Delta p h z \Delta p r n:: l a c Z \Delta v f r$ and its derivatives. (A) Color of colonies shown in the LB medium plate supplemented with X-gal. Arabic numbers from 2 to 7 stand for the fusion mutant G05 $\Delta p h z \Delta p r n:: l a c Z$,

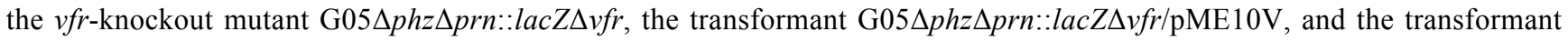
G05 $\Delta$ phz $\Delta p r n:: l a c Z \Delta v f r / p M E 6010$, respectively. (B) $\beta$-Galactosidase activities were quantified when they grown in GA medium at $30^{\circ} \mathrm{C}$ for $72 \mathrm{~h}$. The values from three independent experiments were presented as the average \pm standard deviation. Different superscript lowercase letters followed strains indicate significant difference $(P<0.05)$ according to duncan's multiple range test, and different superscript uppercase letters indicate extremely significant difference $(P<0.01)$. 

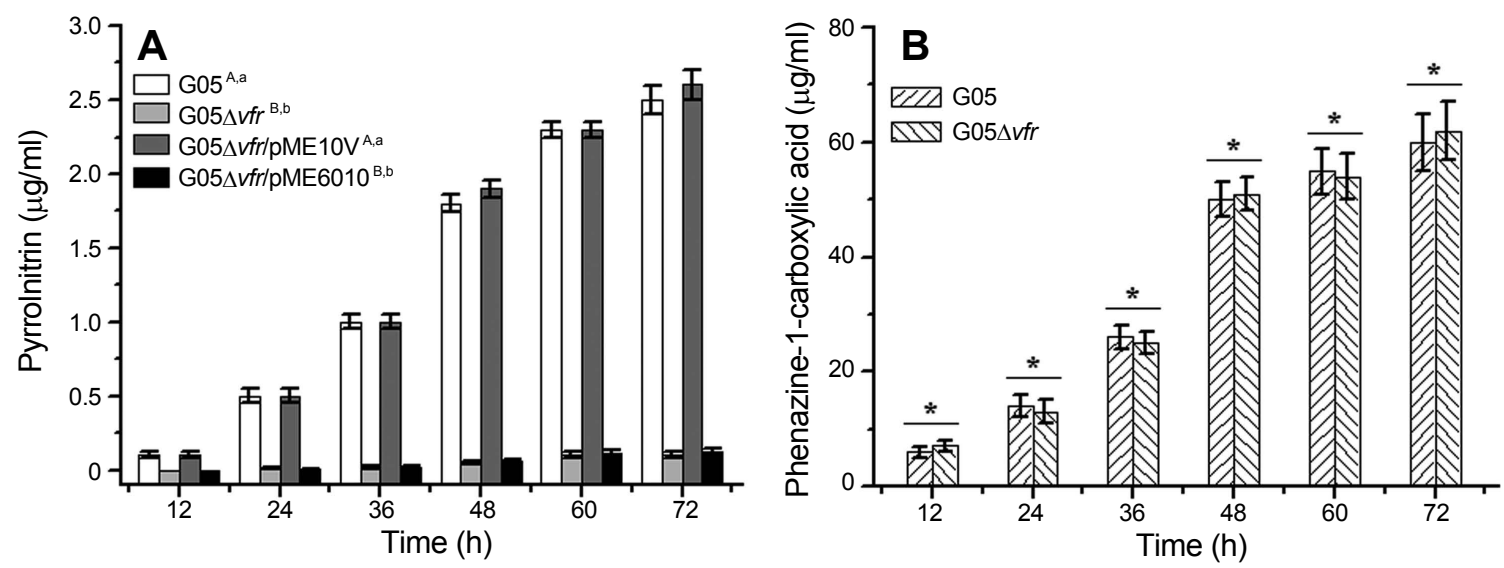

Fig. 3. Regulatory effects of deletion of the $v f r$ on fungal metabolites production in $P$. chlororaphis G05. All experiments were performed in triplicate, and each value was presented as the means \pm standard deviation. (A) Pyrrolnitrin produced by the wild-type strain G05 and its derivatives in GA broth. According to duncan's multiple range test, different superscript lowercase letters followed the strains indicated significant difference $(P<0.05)$, and different superscript uppercase letters followed the strains indicated extremely significant difference $(P<0.01)$. (B) Phenazine-1-carboxylic acid produced by the wild-type strain G05 and its derivatives in GA broth. Asterisks at top of columns mean no significant difference $(P>0.05)$.

Deletion of the $v f r$ brought much less pyrrolnitrin production, but no change of phenazine-1-carboxylic acid. To assess regulatory effects of Vfr on pyrrolnitrin production, we also created the mutant G05 $\Delta v f r$. For quantifying their pyrrolnitrin production, the wild-type strain G05, the mutant G05 $\Delta v f r$ and its derivative transformants were respectively grown in GA medium. As shown in Fig. 3A, in comparison with the wild-type strain G05, the production of pyrrolnitrin in the mutant G05 vvfr was remarkably decreased. When the mutant G05 $\Delta v f r$ was introduced with pME10V, pyrrolnitrin produced in the transformant
G05 $\Delta v \mathrm{fr} / \mathrm{pME10V}$ was almost same to that in the wild-type strain G05. The tranformant G05 $\Delta v f r /$ pME6010, however, looked like its parental strain G05 vvfr and produced a tiny amount of pyrrolnitrin. These results indicated that deletion of the $v f r$ caused much less pyrrolnitrin production in $P$. chlororaphis G05.

In addition, we also determined phenazine-1-carboxylic acid production while they were inoculated and grown in GA medium. According to the Fig. 3B, it was shown that phenazine-1-carboxylic acid produced in the mutant G05 $\Delta v f r$ was same to that in the wild-type strain G05, sug-
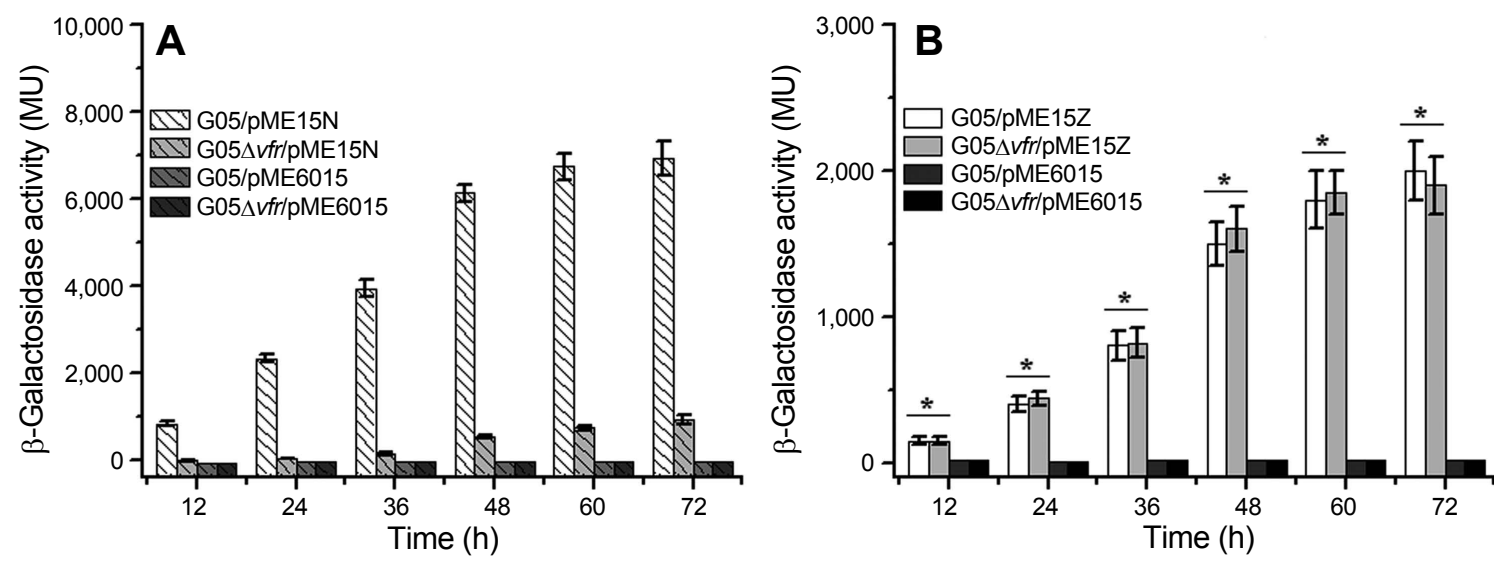

Fig. 4. Translational lacZ fusion vectors pME15Z and pME15N were employed to examine Vfr regulation in P. chlororaphis G05. (A) $\beta$-Galactosidase activities produced by pME15N in the wild-type strain G05 and the mutant G05 $\Delta$ fr were quantified. The transformants G05/pME6015 and G05 $\Delta v \mathrm{fr} / \mathrm{pME6015}$ were used as negative controls. (B) $\beta$-Galactosidase activities produced by pME15Z in the wildtype strain G05 and the mutant G05 $\Delta v f r$ were quantified. The transformants G05/pME6015 and G05 $\Delta v f r / p M E 6015$ were used as negative controls. All experiments were performed in triplicate, and each value was presented as the means \pm standard deviation. Asterisks at top of columns mean no significant difference $(P>0.05)$. 

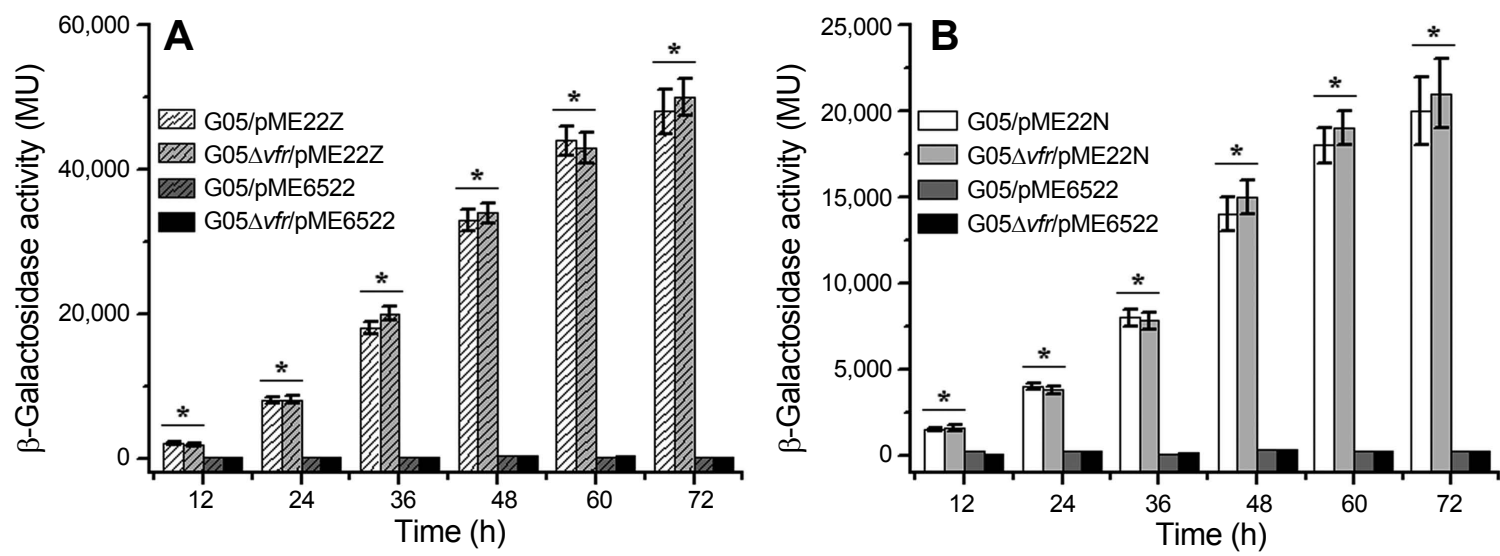

Fig. 5. Translational lacZ fusion vectors pME22Z and pME22N were employed to examine Vfr regulation in P. chlororaphis G05. (A) $\beta$-Galactosidase activities produced by pME22Z in the wild-type strain G05 and the mutant G05 $\Delta$ fr were quantified. The transformant G05/pME6522 and G05 $\Delta v \mathrm{fr} / \mathrm{pME6522}$ were used as negative controls. (B) $\beta$-Galactosidase activities produced by pME22N in the wildtype strain G05 and the mutant G05 $\Delta v f r$ were quantified. The transformant G05/pME6522 and G05 $\Delta v f r / p M E 6522$ were used as negative controls. All experiments were performed in triplicate, and each value was presented as the means \pm standard deviation. Asterisks at top of columns mean no significant difference $(P>0.05)$.

gesting that $\mathrm{Vfr}$ did not exert any effects on the biosynthesis of phenazine-1-carboxylic acid.

Down-regulation of the prn expression mediated by Vfr occurred at the posttranscriptional level, but the phz expression was not regulated by Vfr. To further confirm the results above, we also employed the translational fusions (phzA'-'lacZ and $p r n A^{\prime}-'$ 'lacZ) (Heeb et al., 2000; Zhang et al., 2018) and transcriptional fusions (phzA-lacZ and prnAlacZ) (Blumer et al., 1999; Zhang et al., 2018), did transformation and quantified their $\beta$-galactosidase activities in the wild-type strain $\mathrm{G} 05$ and its derivative mutants. As shown in Fig. 4, $\beta$-galactosidase activities expressed by pME15N (prnA'-'lacZ) in the mutant G05 $\Delta v f r$ were much less than those in the wild-type strain G05. However, $\beta$-galactosidase activities expressed by pME15Z (phz $A^{\prime}-{ }^{\prime}$ lacZ) in the mutant G05 $\Delta v f r$ were almost same to those in the wild-type strain G05. As shown in Fig. 5, $\beta$-galactosidase activities expressed by pME22N (prnA-lacZ) in the mutant G05 $\Delta v f r$ were almost same to those in the wild-type strain G05. Similarly, $\beta$-galactosidase activities expressed by pME22Z (phzA-lacZ) in the mutant G05 $\Delta v f r$ were also same to those in the wild-type strain G05. To verify these results with direct evidences, we also carried out RT-qPCRs to check the transcription of the prnA. As shown in Fig. 6, the copies of mRNA transcribed from the prnA in the mutant G05 vvfr were almost same to those in the wild-type strain G05, confirming that there were no remarkable differences in transcriptional levels of the prn operon in the $v f r$-deletion

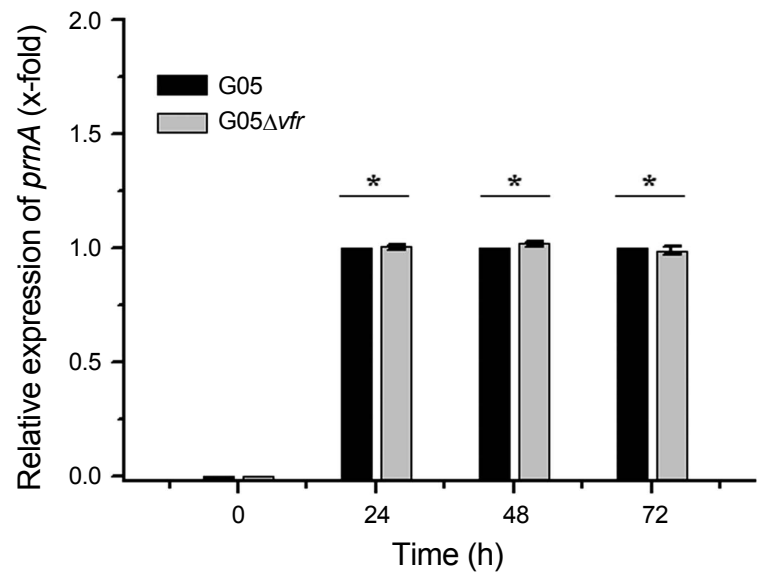

Fig. 6. Gene expression of prnA by RT-qPCR assay in P. chlororaphis $\mathrm{G} 05$ and its derivative mutant G05 $\Delta v f r$. Expression level of the tested prnA in the wild-type strain G05 was considered 1. Relative expressions of prnA in the mutant G05 $\Delta v$ fr compared to the wild-type strain G05 grown in GA medium for $24 \mathrm{~h}, 48 \mathrm{~h}$, and $72 \mathrm{~h}$ were determined by the $2^{-\Delta \Delta \mathrm{CT}}$ method. Asterisks at top of columns mean no significant difference $(P>0.05)$.

mutant G05 $\Delta v f r$ and its parental strain G05. Taken together, no matter whether the $v f r$ gene was mutated with the random transposon insertion or the site-directed deletion in $P$. chlororaphis G05, deficiency of Vfr dramatically downregulated the prn operon expression at the posttranscriptional level, but not at the transcriptional level. Meanwhile, Vfr did not exert any regulatory effects on the $p h z$ expression. 


\section{Disscussion}

As an important global regulator, Vfr first was identified and designated in $P$. aeruginosa duo to its regulatory effects on the biosynthesis of virulence factors (West et al., 1994). In fact, it is a homologue of a transcriptional regulator cyclic AMP receptor protein (Crp) in E. coli, which mediates the expression of more than 100 genes, as well as the biosynthesis of at least 60 proteins (Suh et al., 2002; Wolfgang et al., 2003). Today, a few of homologues of the Crp regulator have been identified in different bacterial genera and their many regulatory effects on virulenceassociated phenotypes have been elucidated, such as iron uptake ability and virulence-host relationships (Taguchi and Ichinose, 2013). In general, Vfr is not only related tightly to the pathogenicity of some bacteria, but also plays a critical role in their infection. In one other previous study, it was reported Vfr in P. fluorescens had a negative regulation on the biosynthesis of secondary antifungal metabolites, such as pyrrolnitrin, PLT, and DAPG (Zhang et al., 2016). Knockout of the $v f r$ gene brought increased production of antifungal compounds. Surprisingly, we happened to find that transposon insertion mutagenesis in the $v f r$ gene in the fusion mutant G05 $\Delta p h z \Delta p r n:: l a c Z$ led to much less $\beta$-galactosidase activities, suggesting that mutation of the $v$ fr could inhibit the biosynthesis of pyrrolnitrin in P. chlororaphis G05. To confirm this hypothesis, we made a site-directed knockout of the $v f r$ gene in the wild-

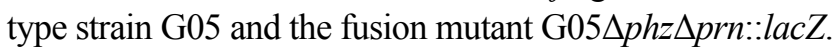
Their pyrrolnitrin production and $\beta$-galactosidase activities verified that deletion of the $v f r$ actually suppressed the expression of the prn operon and biosynthesis of pyrrolnitrin in P. chlororaphis G05. Meanwhile, we also found that Vfr did not exert any regulatory effects on the expression of the phz operon and phenazine-1-carboxylic acid biosynthesis. This is the first report about Vfr-mediated regulation on phenazine production although phenazine biosynthesis is regulated by many well-known regulators (Bilal et al., 2017; Mavrodi et al., 2006). The fact that Vfr differentially regulates two antifungal compounds production in a strain suggests each of two secondary metabolites, pyrrlnitrin and phenazine-1-carboxylic acid, has respectively been synthesized under the control of their own specific regulatory cascade. Obviously, this differential regulation mechanism helps to keep stability of total production of antifungal compounds in the strain G05 and also is helpful in maintaining its biological control function.

Although it has been reported that Vfr could regulate a quite few of metabolites production, the regulation mecha- nism of Vfr has not been elucidated in detail. Using the translational and transcriptional fusions and RT-qPCR, in this study, we tried to understand whether the Vfr-mediated regulation of the prn operon occurs at the transcriptional level or the posttranscriptional level. $\beta$-Galactosidase activities and qPCR indicated that the expression of the prn operon is regulated by Vfr at the posttranscriptional level, not the transcriptional level. Based on these data, we deduced that there might be an intermediate (s) at the downstream of the Vfr-mediated regulatory cascade. This intermediate should be controlled by the Vfr, and in turn, it might directly or indirectly regulate the prn operon expression in $P$. chlororaphis G05. For the detailed Vfr regulation pathway, therefore, further study should be conducted later.

\section{Acknowledgments}

We thank Stephan Heeb (University of Nottingham, Nottingham, the United Kingdom) for providing plasmid pME6015 and pME6522 friendly, Stephen Perle (University of Bridgeport, the Unite States) for his language revision of this manuscript, and Yuquan $\mathrm{Xu}$ (Shanghai Jiaotong University, China) for sending us phenazine-1-carboxylic acid. This study was financially supported by the Natural Science Foundation of China (Grant No. 31260080 and 31571997).

\section{References}

Baehler, E., Bottiglieri, M., Péchy-Tarr, M., Maurhofer, M. and Keel, C. 2005. Use of green fluorescent protein-based reporters to monitor balanced production of antifungal compounds in the biocontrol agent Pseudomonas fluorescens CHA0. J. Appl. Microbiol. 99:24-38.

Bilal, M., Guo, S., Iqbal, H. M. N., Hu, H., Wang, W. and Zhang, X. 2017. Engineering Pseudomonas for phenazine biosynthesis, regulation, and biotechnological applications: a review. World J. Microbiol. Biotechnol. 33:191.

Blumer, C., Heeb, S., Pessi, G. and Haas, D. 1999. Global GacAsteered control of cyanide and exoprotease production in Pseudomonas fluorescens involves specific ribosome binding sites. Proc. Natl. Acad. Sci. U.S.A. 96:14073-14078.

Chen, W. P. and Kuo, T. T. 1993. A simple and rapid method for the preparation of Gram-negative bacterial genomic DNA. Nucleic Acids Res. 21:2260.

Chen, Y., Wang, J., Yang, N., Wen, Z., Sun, X., Chai, Y. and Ma, Z. 2018. Wheat microbiome bacteria can reduce virulence of a plant pathogenic fungus by altering histone acetylation. Nat. Commun. 9:3429.

Chi, X., Wang, Y., Miao, J., Feng, Z., Zhang, H., Zhai, J., Zhang, H., Tian, L., Xue, W., Yang, T., Huang, R., Hu, X. and Ge, Y. 
2017. Development and characterization of a fusion mutant with the truncated lac $Z$ to screen regulatory genes for phenazine biosynthesis in Pseudomonas chlororaphis G05. Biol. Control 108:70-76.

Chieda, Y., Iiyama, K., Yasunaga-Aoki, C., Lee, J. M., Kusakabe, T. and Shimizu, S. 2005. Pathogenicity of gacA mutant of Pseudomonas aeruginosa PAO1 in the silkworm, Bombyx mori. FEMS Microbiol. Lett. 244:181-186.

Cui, Q., Lv, H., Qi, Z., Jiang, B., Xiao, B., Liu, L., Ge, Y. and Hu, X. 2016. Cross-regulation between the $p h z 1$ and $p h z 2$ operons maintains a balanced level of phenazine biosynthesis in Pseudomonas aeruginosa PAO1. PLoS One 11:e0144447.

de Lorenzo, V., Herrero, M., Jakubzik, U. and Timmis, K. N. 1990. Mini-Tn5 transposon derivatives for insertion mutagenesis, promoter probing, and chromosomal insertion of cloned DNA in gram-negative eubacteria. J. Bacteriol. 172:65686572.

D’Mello, J. P. F., Macdonald, A. M. C., Postel, D., Dijksma, W. T. P., Dujardin, A. and Placinta, C. M. 1998. Pesticide use and mycotoxin production in Fusarium and Aspergillus phytopathogens. Eur. J. Plant Pathol. 104:741-751.

Fenton, A. M., Stephens, P. M., Crowley, J., O’Callaghan, M. and O'Gara, F. 1992. Exploitation of gene(s) involved in 2,4-diacetylphloroglucinol biosynthesis to confer a new biocontrol capability to a Pseudomonas strain. Appl. Environ. Microbiol. 58:3873-3878.

Ge, Y., Huang, X., Wang, S., Zhang, X. and Xu, Y. 2004. Phenazine-1-carboxylic acid is negatively regulated and pyoluteorin positively regulated by gacA in Pseudomonas sp. M18. FEMS Microbiol. Lett. 237:41-47.

Ge, Y., Yang, S., Fang, Y., Yang, R., Mou, D., Cui, J. and Wen, L. 2007. RpoS as an intermediate in RsmA-dependent regulation of secondary antifungal metabolites biosynthesis in Pseudomonas sp. M18. FEMS Microbiol. Lett. 268:81-87.

Ge, Y., Chen, L., Wang, L., Su, H., Zhou, J. and Cheng, X. 2008. Effects of insertional inactivation of gacS gene on two secondary metabolites in Pseudomonas chlororaphis G05. Acta. Microbiol. Sinica 48:1595-1601 (in Chinese).

Haas, D. and Défago, G. 2005. Biological control of soil-borne pathogens by fluorescent pseudomonads. Nat. Rev. Microbiol. 3:307-319.

Haas, D. and Keel, C. 2003. Regulation of antibiotic production in root-colonizing Pseudomonas spp. and relevance for biological control of plant disease. Annu. Rev. Phytopathol. 41:117-153.

Heeb, S., Itoh, Y., Nishijyo, T., Schnider, U., Keel, C., Wade, J., Walsh, U., O'Gara, F. and Haas, D. 2000. Small, stable shuttle vectors based on the minimal pVS1 replicon for use in gramnegative, plant-associated bacteria. Mol. Plant-Microbe Interact. 13:232-237.

Hoang, T. T., Karkhoff-Schweizer, R. R., Kutchma, A. J. and Schweizer, H. P. 1998. A broad-host-range Flp-FRT recombination system for site-specific excision of chromosomally-located DNA sequences: application for isolation of unmarked
Pseudomonas aeruginosa mutants. Gene 212:77-86.

Huang, R., Feng, Z., Chi, X., Sun, X., Lu, Y., Zhang, B., Lu, R., Luo, W., Wang, Y., Miao, J. and Ge, Y. 2018. Pyrrolnitrin is more essential than phenazines for Pseudomonas chlororaphis G05 in its suppression of Fusarium graminearum. Microbiol. Res. 215:55-64.

Kim, K. J. 2000. Phenazine 1-carboxylic acid resistance in phenazine 1-carboxylic acid producing Bacillus sp. B-6. J. Biochem. Mol. Biol. 33:332-336.

Laville, J., Voisard, C., Keel, C., Maurhofer, M., Défago, G. and Haas, D. 1992. Global control in Pseudomonas fluorescens mediating antibiotic synthesis and suppression of black root rot of tobacco. Proc. Natl. Acad. Sci. U.S.A. 89:1562-1566.

Liu, Y., Wang, Z., Bilal, M., Hu, H., Wang, W., Huang, X., Peng, H. and Zhang, X. 2018. Enhanced fluorescent siderophore biosynthesis and loss of phenazine-1-carboxamide in phenotypic variant of Pseudomonas chlororaphis HT66. Front. Microbiol. 9:759.

Luo, W., Miao, J., Feng, Z., Lu, R., Sun, X., Zhang, B., Ding, W., Lu, Y., Wang, Y., Chi, X. and Ge, Y. 2019. Construction of a $\beta$-galactosidase-gene-based fusion is convenient for screening candidate genes involved in regulation of pyrrolnitrin biosynthesis in Pseudomonas chlororaphis G05. J. Gen. Appl. Microbiol. 64:259-268.

Mavrodi, D. V., Ksenzenko, V. N., Bonsall, R. F., Cook, R. J., Boronin, A. M. and Thomashow, L. S. 1998. A seven-gene locus for synthesis of phenazine-1-carboxylic acid by Pseudomonas fluorescens 2-79. J. Bacteriol. 180:2541-2548.

Mavrodi, D. V., Blankenfeldt, W. and Thomashow, L. S. 2006. Phenazine compounds in fluorescent Pseudomonas spp. biosynthesis and regulation. Annu. Rev. Phytopathol. 44:417445.

Miller, J. H. 1972. Experiments in molecular genetics. Cold Spring Harbor Laboratory Press, Cold Spring Harbor, NY, USA. 466 pp.

Minton, N. P. 1984. Improved plasmid vectors for the isolation of translational lac gene fusions. Gene 31:269-273.

Mulet, M., Bennasar, A., Lalucat, J. and García-Valdés, E. 2009. An rpoD-based PCR procedure for the identification of Pseudomonas species and for their detection in environmental samples. Mol. Cell. Probes 23:140-147.

Livak, K. J. and Schmittgen, T. D. 2001. Analysis of relative gene expression data using real-time quantitative PCR and the $2(-\Delta \Delta \mathrm{C}(\mathrm{T}))$ method. Methods 25:402-408.

Nandi, M., Selin, C., Brawerman, G., Fernando, W. G. and de Kievit, T. R. 2016. The global regulator ANR is essential for Pseudomonas chlororaphis strain PA23 biocontrol. Microbiology 162:2159-2169.

Oh, S. A., Kim, J. S., Park, J. Y., Han, S. H., Dimkpa, C., Anderson, A. J. and Kim, Y. C. 2013. The RpoS sigma factor negatively regulates production of IAA and siderophore in a biocontrol Rhizobacterium, Pseudomonas chlororaphis O6. Plant Pathol. J. 29:323-329.

Ovadis, M., Liu, X., Gavriel, S., Ismailov, Z., Chet, I. and 
Chernin, L. 2004. The global regulator genes from biocontrol strain Serratia plymuthica IC1270: cloning, sequencing, and functional studies. J. Bacteriol. 186:4986-4993.

Park, J. Y., Kang, B. R., Ryu, C. M., Anderson, A. J. and Kim, Y. C. 2018. Polyamine is a critical determinant of Pseudomonas chlororaphis $\mathrm{O} 6$ for GacS-dependent bacterial cell growth and biocontrol capacity. Mol. Plant Pathol. 19:1257-1266.

Sambrook, J. and Russell, D. W. 2001. Molecular cloning: A laboratory manual. 3rd ed. Cold Spring Harbor Laboratory Press, Cold Spring Harbor, NY, USA. 2100 pp.

Schweizer, H. D. 1993. Small broad-host-range gentamycin resistance cassettes for site-specific insertion and deletion mutagenesis. BioTechniques 15:831-834.

Shah, N., Klaponski, N., Selin, C., Rudney, R., Fernando, W. G., Belmonte, M. F. and de Kievit, T. R. 2016. PtrA is functionally intertwined with GacS in regulating the biocontrol activity of Pseudomonas chlororaphis PA23. Front. Microbiol. 7:1512.

Smith, A. W. and Iglewski, B. H. 1989. Transformation of Pseudomonas aeruginosa by electroporation. Nucleic Acids Res. 17:10509.

Suh, S. J., Runyen-Janecky, L. J., Maleniak, T. C., Hager, P., MacGregor, C. H., Zielinski-Mozny, N. A., Phibbs, P. V. and West, S. E. 2002. Effect of vfr mutation on global gene expression and catabolite repression control of Pseudomonas aeruginosa. Microbiology 148:1561-1569.

Taguchi, F. and Ichinose, Y. 2013. Virulence factor regulator (Vfr) controls virulence-associated phenotypes in Pseudomonas syringae pv. tabaci 6605 by a quorum sensing-independent mechanism. Mol. Plant Pathol. 14:279-292.

Thomashow, L. S. and Weller, D. M. 1988. Role of a phenazine antibiotic from Pseudomonas fluorescens in biological control of Gaeumannomyces graminis var. tritici. J. Bacteriol. 170:3499-3508.

Trippe, K., McPhail, K., Armstrong, D., Azevedo, M. and Banowetz, G. 2013. Pseudomonas fluorescens SBW25 produces furanomycin, a non-proteinogenic amino acid with selective antimicrobial properties. BMC Microbiol. 13:111.

Voisard, C., Keel, C., Haas, D. and Dèfago, G. 1989. Cyanide production by Pseudomonas fluorescens helps suppress black root rot of tobacco under gnotobiotic conditions. EMBO J. 8:351-358.

West, S. E., Sample, A. K. and Runyen-Janecky, L. J. 1994. The vfr gene product, required for Pseudomonas aeruginosa exotoxin A and protease production, belongs to the cyclic AMP receptor protein family. J. Bacteriol. 176:7532-7542.

Wolfgang, M. C., Lee, V. T., Gilmore, M. E. and Lory, S. 2003. Coordinate regulation of bacterial virulence genes by a novel adenylate cyclase-dependent signaling pathway. Dev. Cell 4:253-263.

Zhang, Q., Xiao, Q., Xu, J., Tong, Y., Wen, J., Chen, X. and Wei, L. 2015. Effect of retS gene on antibiotics production in Pseudomonas fluorescens FD6. Microbiol. Res. 180:23-29.

Zhang, Q., Ji, Y., Xiao, Q., Chng, S., Tong, Y., Chen, X. and Liu, F. 2016. Role of Vfr in the regulation of antifungal compound production by Pseudomonas fluorescens FD6. Microbiol. Res. 188-189:106-112.

Zhang, B., Wang, Y., Miao, J., Lu, Y., Lu, R., Sun, X., Luo, W., Chi, X., Feng, Z. and Ge, Y. 2018. Reciprocal enhancement of gene expression between the phz and prn operon in Pseudomonas chlororaphis G05. J. Basic Microbiol. 58:793-805. 